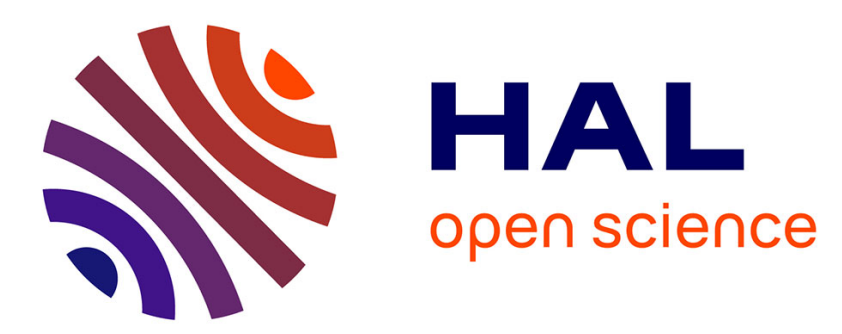

\title{
Application of the generalized lambda distributions in a statistical process control methodology
}

Benjamin Fournier, Nicolas Rupin, Maxence Bigerelle, Denis Najjar, Alain Iost

\section{To cite this version:}

Benjamin Fournier, Nicolas Rupin, Maxence Bigerelle, Denis Najjar, Alain Iost. Application of the generalized lambda distributions in a statistical process control methodology. Journal of Process Control, 2006, 16 (10), pp.1087-1098. 10.1016/j.jprocont.2006.06.009 . hal-01315201

\author{
HAL Id: hal-01315201 \\ https://hal.science/hal-01315201
}

Submitted on 12 May 2016

HAL is a multi-disciplinary open access archive for the deposit and dissemination of scientific research documents, whether they are published or not. The documents may come from teaching and research institutions in France or abroad, or from public or private research centers.
L'archive ouverte pluridisciplinaire HAL, est destinée au dépôt et à la diffusion de documents scientifiques de niveau recherche, publiés ou non, émanant des établissements d'enseignement et de recherche français ou étrangers, des laboratoires publics ou privés. 


\title{
Application of the generalized lambda distributions in a statistical process control methodology
}

\author{
B. Fournier ${ }^{\mathrm{a}, *}$, N. Rupin ${ }^{\mathrm{a}, 1}$, M. Bigerelle ${ }^{\mathrm{a}, \mathrm{b}}$, D. Najjar ${ }^{\mathrm{a}}$, A. Iost ${ }^{\mathrm{a}}$ \\ ${ }^{a}$ LMPGM Laboratoire de Métallurgie Physique et Génie des Matériaux, CNRS UMR 8517, Caractérisation et Propriétés de la Périsurface, \\ ENSAM, 8 Boulevard Louis XIV, 59046 Lille cedex, France \\ ${ }^{\mathrm{b}}$ Laboratoire Roberval, FRE 2833, UTC/CNRS, Centre de Recherches de Royallieu, BP20529, 60205 Compiègne, France
}

\begin{abstract}
In statistical process control (SPC) methodology, quantitative standard control charts are often based on the assumption that the observations are normally distributed. In practice, normality can fail and consequently the determination of assignable causes may result in error. After pointing out the limitations of hypothesis testing methodology commonly used for discriminating between Gaussian and non-Gaussian populations, a very flexible family of statistical distributions is presented in this paper and proposed to be introduced in SPC methodology: the generalized lambda distributions (GLD). It is shown that the control limits usually considered in SPC are accurately predicted when modelling usual statistical laws by means of these distributions. Besides, simulation results reveal that an acceptable accuracy is obtained even for a rather reduced number of initial observations (approximately a hundred). Finally, a specific user-friendly software have been used to process, using the SPC Western Electric rules, experimental data originating from an industrial production line. This example and the fact that it enables us to avoid choosing an a priori statistical law emphasize the relevance of using the GLD in SPC.
\end{abstract}

Keywords: Statistical process control; Western electric rules; Hypothesis testing; Generalized lambda distributions; Numerical simulations; Non-normality; Sampling data

\section{Introduction}

The statistical process control (SPC) methodology is mainly used as a tool to understand, model and reduce the variability of an industrial process over time. It is based on the detection of "non-random" patterns on control charts; many of these patterns being reported in the Western Electric Handbook [1] which defines the eight main detection rules reminded in Appendix. Usually based on

\footnotetext{
${ }^{*}$ Corresponding author. Present address: CEA/DEN-DANS/DMN/ SRMA/LC2M, Bat. 455, 91191 Gif-sur-Yvette Cedex, France. Tel.: +33 3 206222 33; fax: +33 320622957 .

E-mail address: benjamin.fournier@cea.fr (B. Fournier).

${ }^{1}$ Present address: Solid Mechanics Laboratory, CNRS UMR 7649, Department of Mechanics, Ecole Polytechnique, 91128 Palaiseau, Cedex, France.
}

graphical tools such as control charts, the SPC methodology uses inference statistical analysis of monitored time series data to distinguish between common causes of variation due to the inherent nature of the process and assignable causes due to unusual shocks or disruption. Lots of quantitative control charts exist today and their use is generally based on the assumption that the distribution of the process data under study can be modelled by one of the usual probability laws (Gaussian, lognormal, Weibull,...).

In SPC strategy, the most widely used assumption is that the population under study follows a Gaussian law. It is often justified by the fact that any measure presents a variability due to many independent causes. In fact, the superposition of all these independent causes (having compensative influences) leads to a distribution of the variable under consideration that converges to a Gaussian law. This simplistic explanation results from the well known Central 
Limit Theorem [2] which states that the summation of an infinite number of randomly distributed variables converges to a normal distribution. Nevertheless, several practical examples of non-Gaussian processes were often reported [3-6]. Whether the hypothesized model is Gaussian or not is assessed by means of a hypothesis test. However, whatever the statistical test a major drawback must be outlined. Indeed, the results of such tests only warranty the eventual rejection of the classical null hypothesis $\mathrm{H}_{0}$ (The data under study follow the hypothesized law). Conversely, failure to reject does not necessarily mean that the tested null hypothesis is true and it would be very interesting to get through this limitation of statistical inference.

The present work aims to present an alternative statistical approach that avoids the previously cited drawback related to statistical inference. In this approach, a very flexible family of distributions called the GLD is used to model accurately a series of data while minimizing hypotheses about the nature and the shape of the unknown underlying distribution. These distributions have been applied by the authors firstly to predict the fatigue lifetime of metals [7] and to model extreme values during the pitting corrosion process [8]. Two years ago the authors introduced the use of the GLD in the SPC methodology [9] and in a very recent paper [10], Pal used the lambda distributions to calculate the capability indices ( $\mathrm{CP}$ and $\mathrm{CPK})$ widely used in statistical process control.

In the present paper, a major limitation of the classical $\chi^{2}$ goodness-of-fit test is firstly pointed out from the analysis of a simple example: in practice, a large amount of data must be processed to reject the assumption of normality. Secondly, the GLD are presented and used to model Gaussian, lognormal and Weibull data in order to show that they are particularly well-adapted to approach lots of various shapes of distributions and also to predict accurately the control limits considered in SPC methodology. A numerical simulation is performed to evaluate in particular the minimal number of data required for an accurate prediction of these limits when considering a GLD based modelling. Finally, specific user-friendly software, are used to process experimental data originating from an industrial production line and to emphasize the relevance of considering GLD-based modelling in SPC.

\section{Limitations of the Gaussian assumption}

A simple illustrated example is developed below on data following distributions far from being Gaussian in order to show that a high number of values are required to reject the null hypothesis $\mathrm{H}_{0}$ ("The data under study are normaly distributed") studied by means of a classical goodness-of-fit test. In this example, distributions have been obtained by summing uniform variables generated by means of Monte-Carlo simulations. For the sake of simplicity, these distributions will be denoted, $F_{N}$, with $N$ being the number of summated uniform variables hereafter called the "degree of summation". Thus, a set of $10^{6}$ uniform scores has firstly been generated to obtain a classical uniform distribution $(N=1)$. Secondly, two uniform variables have been generated and their sum has been calculated. When repeating $10^{6}$ times these operations, the resulting sum follows a usual triangular distribution $(N=2)$. Thirdly, three uniform variables have been generated and their sum has been calculated. When repeating $10^{6}$ times these operations, the resulting sum follows in this case a distribution which consists of three pieces of parabola $(N=3)$.

For these distributions far from being Gaussian, the question is therefore to determine how many values are required to reject the null hypothesis $\mathrm{H}_{0}$ ("The data under study are normaly distributed") by means of the most widely used $\chi^{2}$ goodness-of-fit test. To evaluate this minimal number of values for a given value of $N$, datasets of $n$ values following the law $F_{N}$ are generated. Then, the $p$ value representing the probability to reject falsely $\mathrm{H}_{0}$ is estimated by means of the $\chi^{2}$ goodness-of-fit test. This process is repeated $10^{5}$ times and the mean of $p$-values is calculated. The evolution of the mean $p$-values is represented in Fig. 1 as a function of the sample size $n$ for the distributions $F_{N}$ for $N=1-5$; each point representing the mean of $p$-values calculated for a $10^{5}$ samples with the same size $n$. In practice, a critical probability $\alpha$ is often selected to specify an acceptable Type I error (occurring if the null hypothesis $\mathrm{H}_{0}$ is falsely rejected). The classical value $\alpha=0.05$ is usually retained, meaning that the user accepts to reject falsely the distribution's normality in 5\% of cases. For this classical value, Fig. 1 shows that, even for simulated data originating from distributions far from being Gaussian, a significant number of values is required so that the $\chi^{2}$ goodness-of-fit test rejects the normality assumption: at least 30 values in the case of a uniform distribution $(N=1)$ and more than 500 values in the case of a triangular distribution $(N=2)$ !

Fig. 2 shows that the minimal number of values required to reject $\mathrm{H}_{0}$ at a critical value $\alpha=0.05$ exponentially increases as a function of the "degree of summation" $N$; i.e. as the distribution under consideration tends to the true Gaussian distribution (obtained when $N \rightarrow+\infty$ as stated by the Central Limit Theorem). For $N=3$, this minimal value exceeds 2500 data showing that, in comparison with distributions that are far from being Gaussian, it is even more difficult to reject the Gaussian assumption using this classical $\chi^{2}$ goodness-of-fit test in the case of distributions simply looking similar (without however being precisely equal) to the true Gaussian distribution. Unfortunately, such a high number of experimental data is rarely processed in practice, meaning that, using a classical hypothesis test, the Gaussian law may be abusively accepted in SPC to model the unknown underlying population even if it does not obey a true Gaussian law!

As far as the SPC methodology is concerned, the Western Electric rules [1] are based on the comparison of the position of data under processing with regard to the limits of the confidence intervals of the Gaussian distribution. Fig. 3a represents the estimated width of the $99.73 \%$ 


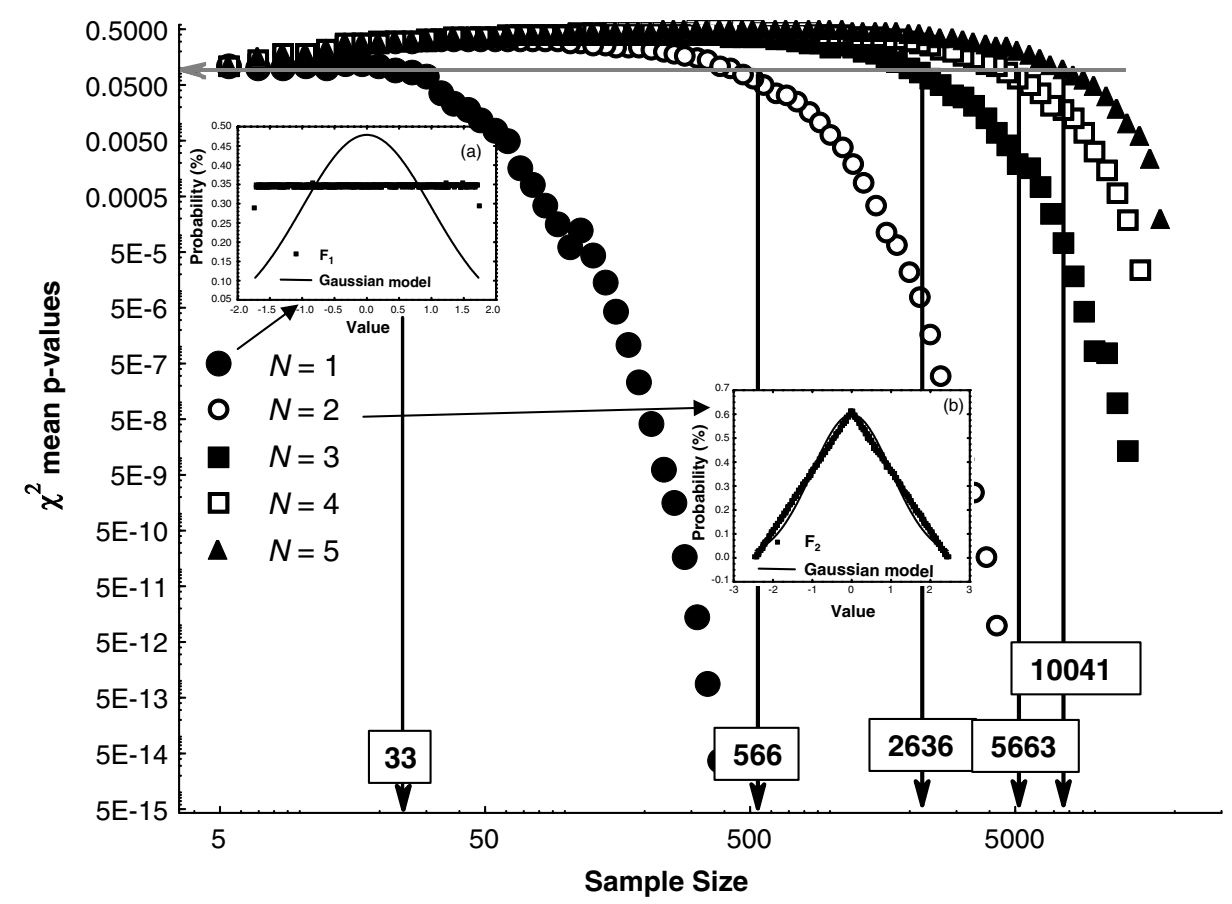

Fig. 1. Evolution of the $\chi^{2}$ mean $p$-values as a function of the sample size $n$ for a degree of summation $N$ varying from 1 to 5 . The corresponding distributions $F_{N}$ are inserted in windows as well as the result of their Gaussian modelling for $N=1$ and $N=2$.

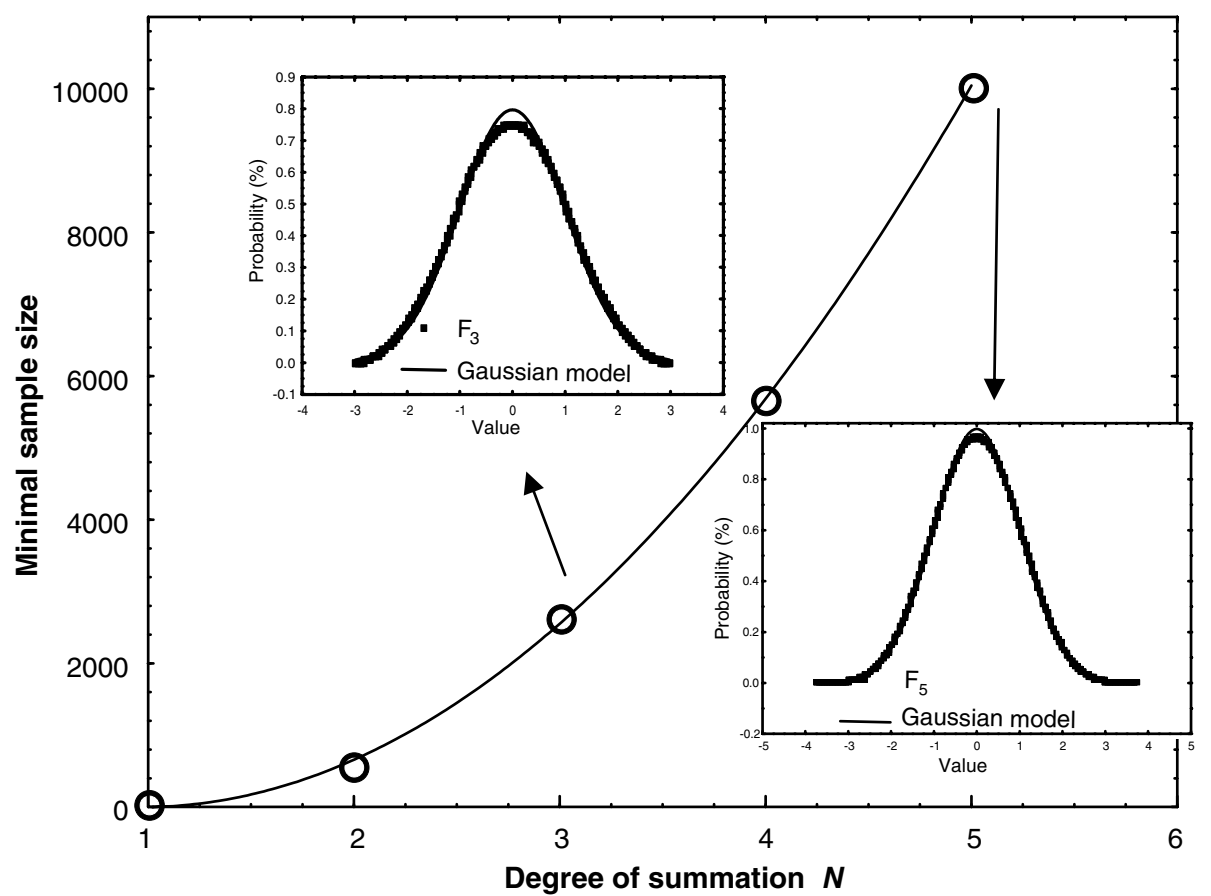

Fig. 2. Evolution of the minimal sample size required to reject $\mathrm{H}_{0}$ with a Type I error $\alpha=0.05$ as a function of the degree of summation $N$. The distributions $F_{N}$ are inserted in windows as well as the result of their Gaussian modelling for $N=3$ and $N=5$.

confidence interval of simulated data, for a degree of summation $N$ varying from 1 to 50 . It can be seen that this width increases with the value of $N$ and that it tends towards $6 \sigma$; value corresponding to the true Gaussian distribution $(N \rightarrow+\infty)$. As a consequence, if a $6 \sigma$ confidence interval is set as recommended in the Western Electric rules based on the Gaussian assumption, the probability to find a simulated value out of this interval is all the lower as the value of $N$ is also small. Fig. $3 \mathrm{~b}$ shows that this probability tends to $0.27 \%$ as the degree of summation increases; 


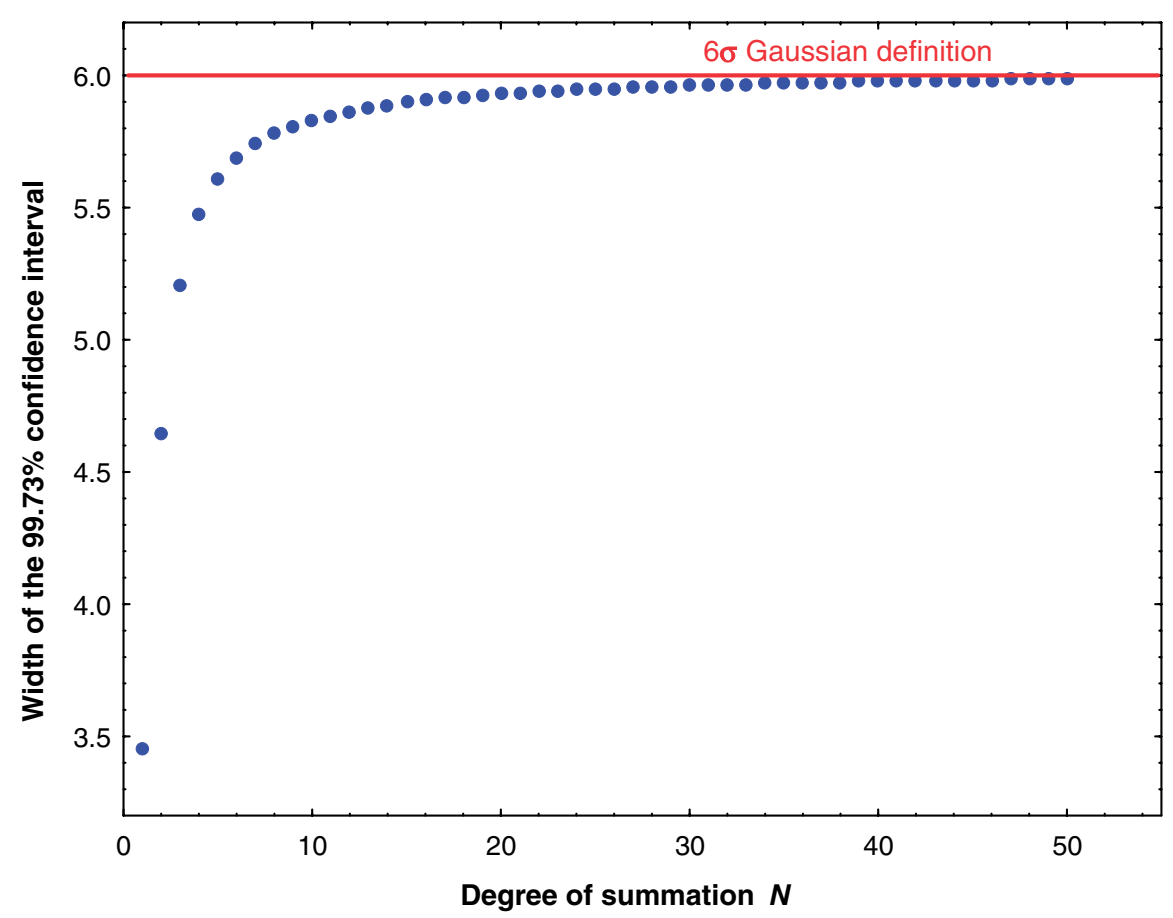

Fig. 3a. Evolution of the $99.73 \%$ confidence interval width (in terms of units of standard deviation $\sigma$ ) of simulated data as a function of the degree of summation $N$.

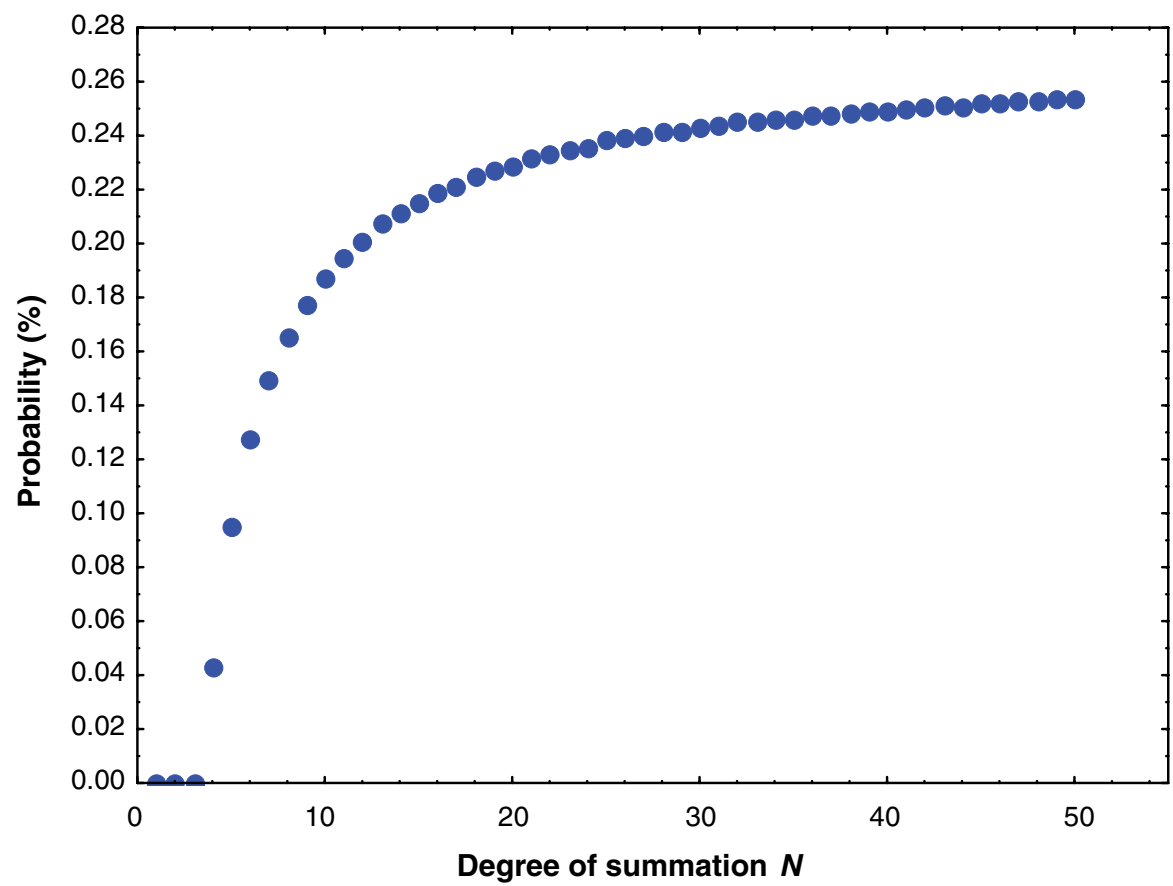

Fig. 3b. Evolution of the probability to find a simulated data out of the Gaussian $6 \sigma$ confidence interval as a function of the degree of summation $N$.

expected value in the case of a Gaussian distribution. For $N=1$ (uniform), $N=2$ (triangular) and $N=3$ (pieces of parabola), this probability becomes null. For these distributions having small $N$ values, the first and major Western Electric rule [1] (one point out of the $6 \sigma$ interval) is simply useless... This is another striking illustration of the incidences of abusive Gaussian modelling due to a lack of experimental data.

There is no denying that other statistical tests could be used in combination to the usual $\chi^{2}$ goodness-of-fit test 
to assess the validity of the Gaussian assumption. A simple alternative test would be to check whether the distribution under study is symmetric or not. To do so, numerous tests are available [11-15]. Nevertheless, if such complementary tests can help to reject the Gaussian assumption (and then to choose a more appropriate distribution), their power as in the case of the $\chi^{2}$ goodness-of-fit test remains linked to the number of available data.

The previous examples, that illustrated such limitations due to the abusive use of the Gaussian assumption in the SPC methodology, are the major reasons for considering an alternative methodology which avoids making any assumption about the unknown underlying distribution. The methodology we proposed is based on the use of a very flexible and attractive family of statistical distributions called the generalized lambda distributions.

\section{The generalized lambda distributions}

In process control strategies, accurate modelling of the process probability density function (PDF) must be used. This is pointed out by Forbes et al. [16], who used a Gram-Charlier PDF to obtain a standard state-space model of the process. In the framework of dynamic processes, Wang [17] proposed a general time-evolving model of the process PDF based on B-splines. Several other attempts have been made to find a family of statistical distributions that could accurately model a broad class of distribution shapes. For example, Albers et al. [18] used the normal power family, Lin and Chou [19] the Burr distribution and Johnson [20] introduced the use of Johnson's family in capability analysis. Defined by means of four parameters, the GLD firstly introduced by Tukey [21]

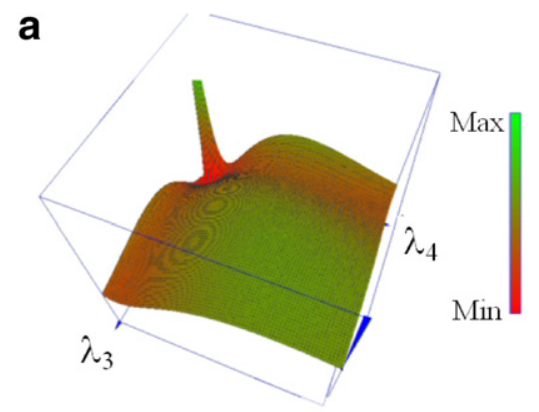

b

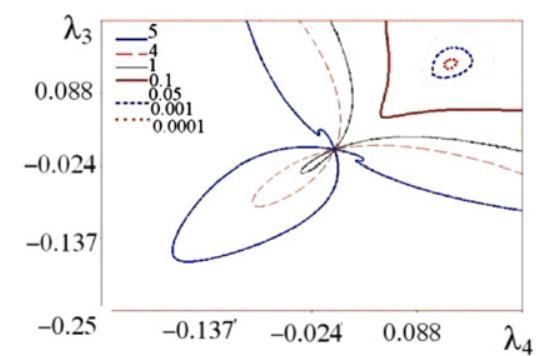

Fig. 4. Example of the 3D visualisation of a surface $S\left(\lambda_{3}, \lambda_{4}\right)$ (a) and its related contour plot (b) obtained by means of the LambdaFinder software.
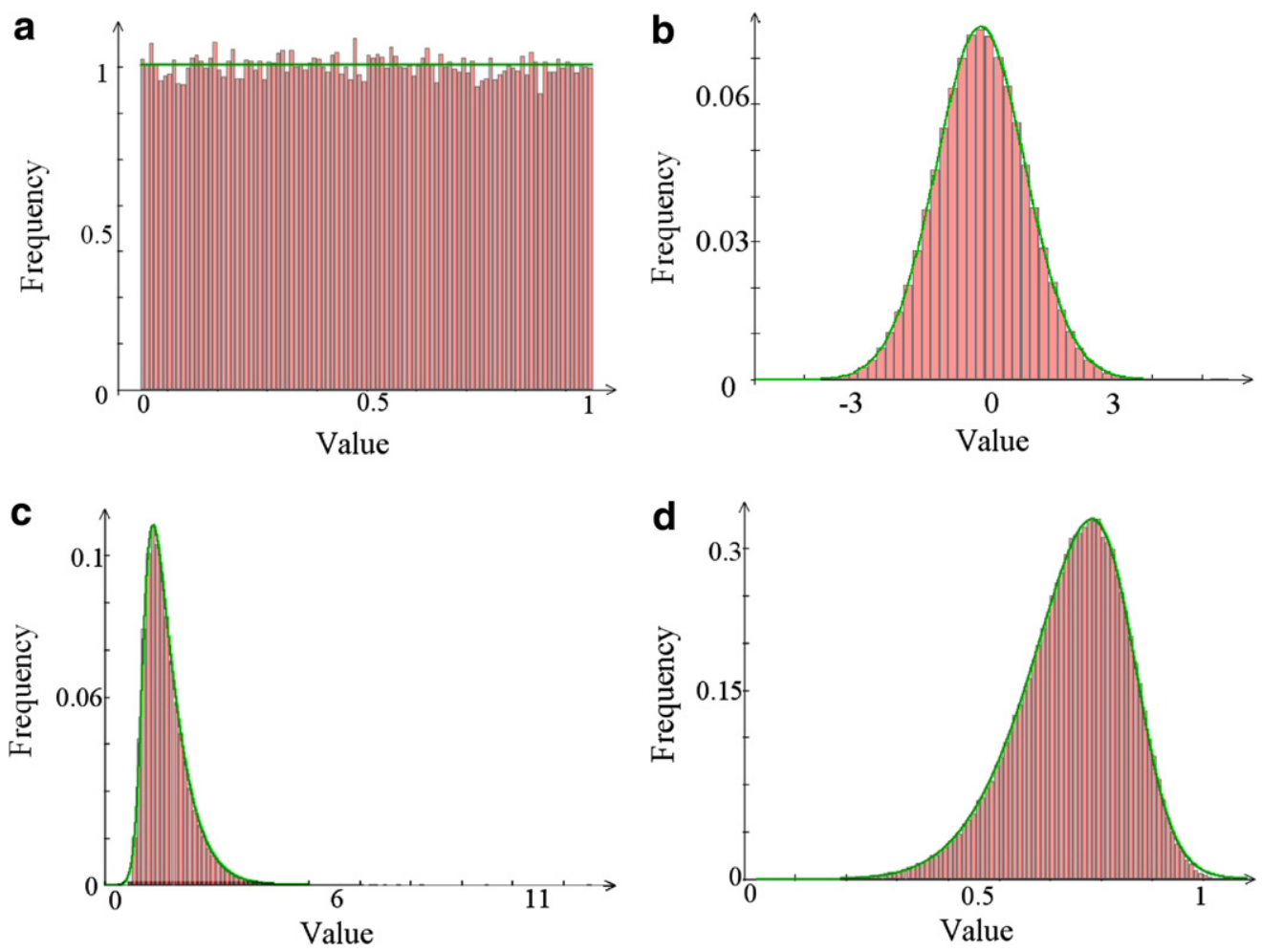

Fig. 5. Generalized lambda distribution based modelling results obtained using the LambdaFinder software in the cases of (a) an uniform distribution, (b) a Gaussian distribution, (c) a lognormal distribution and (d) a right skewed Weibull distribution. 
and further described by others [22-25] have already been shown to fit accurately most of the usual statistical distributions (Gaussian, lognormal, Weibull, uniform,...). Due to this ability the GLD have been used in lots of various scientific fields, such as chemical process monitoring [26,27], finance [28,29], corrosion [8] or meteorology [30]... Such a flexibility with regard to the shape of many distributions enables to consider the GLD in a more global SPC strategy without making any preconceived and risky choice about the unknown underlying distribution.

\subsection{Definition}

In this paragraph, the definition of this family of distributions is briefly reminded. For more details, the reader should refer to the recent monograph written by Karian and Dudewicz [31].

A GLD, noted GLD $\left(\lambda_{1}, \lambda_{2}, \lambda_{3}, \lambda_{4}\right)$, could be specified firstly by its percentile function defined with parameters $\left(\lambda_{1}, \lambda_{2}, \lambda_{3}, \lambda_{4}\right)$ :

$Q(y)=Q\left(y ; \lambda_{1}, \lambda_{2}, \lambda_{3}, \lambda_{4}\right)=\lambda_{1}+\frac{y^{\lambda_{3}}-(1-y)^{\lambda_{4}}}{\lambda_{2}}$

with $y \in[0,1],\left(\lambda_{1}, \lambda_{2}\right)$ are respectively the position and scale parameters and $\left(\lambda_{3}, \lambda_{4}\right)$ are respectively related to the skewness (asymmetry) and the kurtosis (flatness) of the GLD $\left(\lambda_{1}, \lambda_{2}, \lambda_{3}, \lambda_{4}\right)$.

It must be pointed out that this percentile-based definition for a GLD is very interesting with a view to running subsequent easy and fast Monte-Carlo simulations. Besides, the related PDF of the GLD $\left(\lambda_{1}, \lambda_{2}, \lambda_{3}, \lambda_{4}\right)$ can eas-
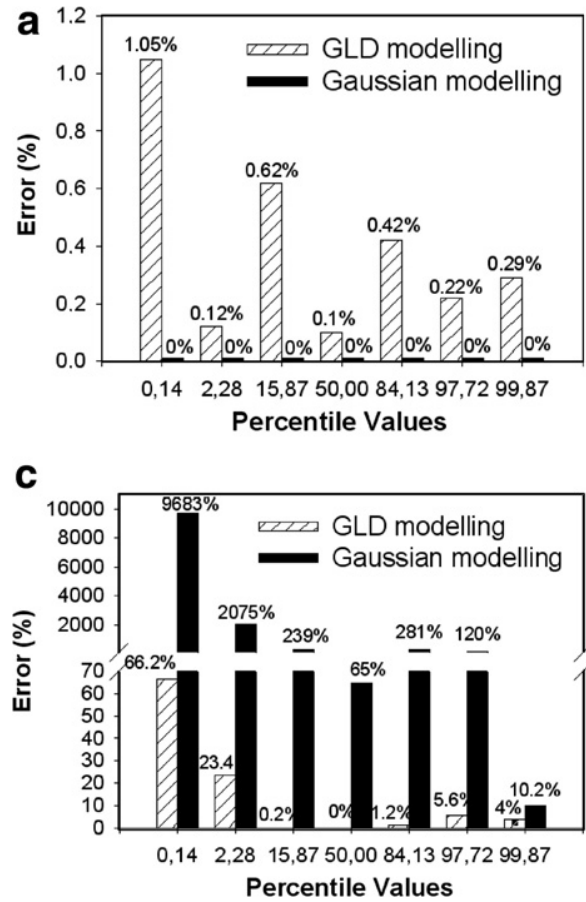

ily be derived from the equation of the above percentile function:

$f(x)=f(Q(y))=\frac{\lambda_{2}}{\lambda_{3} y^{\lambda_{3}-1}+\lambda_{4}(1-y)^{\lambda_{4}-1}}$

\subsection{Determination of the $G L D$}

As pointed out by Karian and Dudewicz [31,32], the calculation of the values of the parameters $\left(\lambda_{1}, \lambda_{2}, \lambda_{3}, \lambda_{4}\right)$ for which the $\operatorname{GLD}\left(\lambda_{1}, \lambda_{2}, \lambda_{3}, \lambda_{4}\right)$ models the most accurately the histogram of experimental or simulated values that characterizes the population under study is not an easy task. The two most usual methods are the method of moments and the method of percentiles [31]. Roughly speaking, both these methods consist in minimizing a relatively complex function presenting several local minima (Fig. 4). To perform this special task we resorted to a computer program called LambdaFinder. This software, we developed two years ago [9] was particularly intended to provide a three dimensional visualisation of the surface and of its local minima [33].

\section{Introduction of GLD in SPC methodology}

\subsection{Modelling of usual laws}

As already mentioned, thanks to their definition based on four parameters, the main advantage of the GLD is their ability to model accurately a large panel of unimodal distributions. Such attractive property is illustrated in
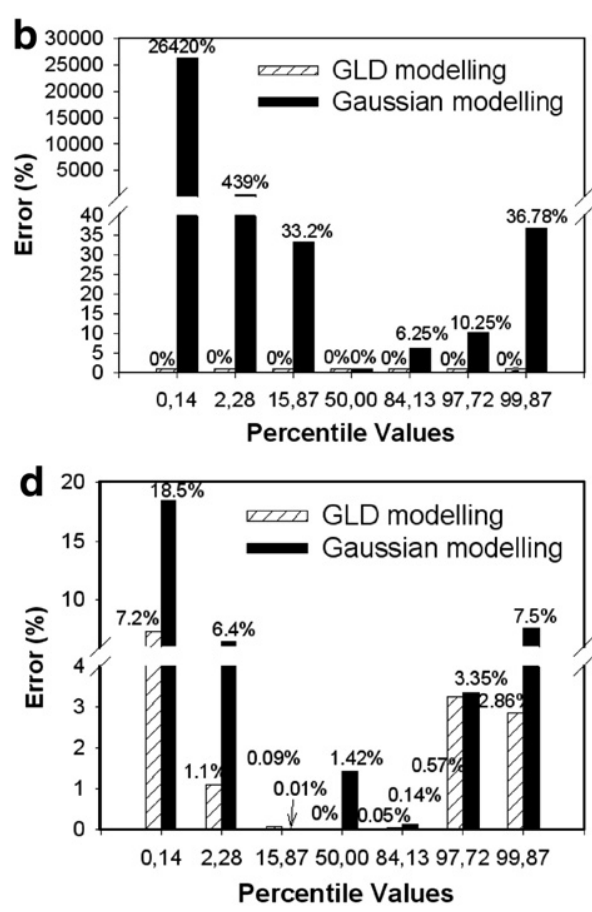

Fig. 6. Comparison of the accuracy of SPC limits predicted in the case of both Gaussian and GLD-based modelling for data generated from (a) a Gaussian distribution, (b) an uniform distribution, (c) a lognormal distribution and (d) a Weibull distribution. Error (\%) is defined as: Error $(\%)=\left|\frac{\text { Theoretical value-Predicted value }}{\text { Theoretical value }}\right| \times 100$. 
Fig. 5 for usual distributions like uniform, Gaussian, lognormal or Weibull distributions for which $10^{7}$ values have been simulated before being processed by the LambdaFinder software. Then, these four usual distributions have also been modelled using Gaussian distributions to simulate the errors that can be made during a SPC process falsely based on the most widely used normality assumption. Indeed, as previously mentioned, a reduced initial amount of experimental data in practice may lead to accept abusively the Gaussian law for modelling the unknown underlying population even if it is heavily skewed and does not obey a Gaussian law.

The values of the percentiles used in the definition of the classical SPC control confidence intervals and the value of 50 th percentile used to estimate the central tendency have been determined for each of the four usual distributions modelled using either Gaussian distribution or GLD. The obtained values, presented in Fig. 6, result from the processing of a large number of simulated data $\left(10^{7}\right)$ so that the estimated experimental percentiles can be considered as equal to their "true values". These results show that the GLD allow to model all the statistical laws under study and, except in the obvious case for which data precisely originate from a Gaussian law, to predict also more accurately the limits of their confidence intervals in comparison with a Gaussian-based modelling. In the case of GLDbased modelling of data originating from a Gaussian law, the maximal error is only $1 \%$ and the central tendency is estimated with a precision of $0.1 \%$, which is widely sufficient in most practical cases.

Besides, it can be seen that, contrary to the Gaussian distribution, the GLD can perfectly model data originating from a uniform law since every error on the estimated percentiles is null. However, the major advantage of GLDbased modelling is emphasized in the case of the analysis of data originating from common skewed distributions like lognormal or Weibull ones. Indeed, for these distributions, the SPC intervals defined in terms of percentiles are asymmetrical (i.e. they are not centered on the distribution mean contrary to that resulting from a Gaussian based modelling). In the case of GLD-based modelling, the limits calculated for these intervals result from the rigorous definition of the related percentile function. As a consequence, the precision obtained on the predicted limits is always superior or in worse cases noticeably equal to that resulting from Gaussian-based modelling.

In summary, these results show that, for all studied distributions, the GLD allow more flexible modelling of shapes than the classical Gaussian law while keeping a very accurate approximation of the predicted limits considered in SPC methodology. That is why such a family of distributions should be considered with particular attention as far as SPC methodology is concerned. Nevertheless there is no denying that the GLD cannot model all the possible shapes of statistical distribution, and that the adequacy with the data under study must be assessed by performing goodness-of-fit tests.
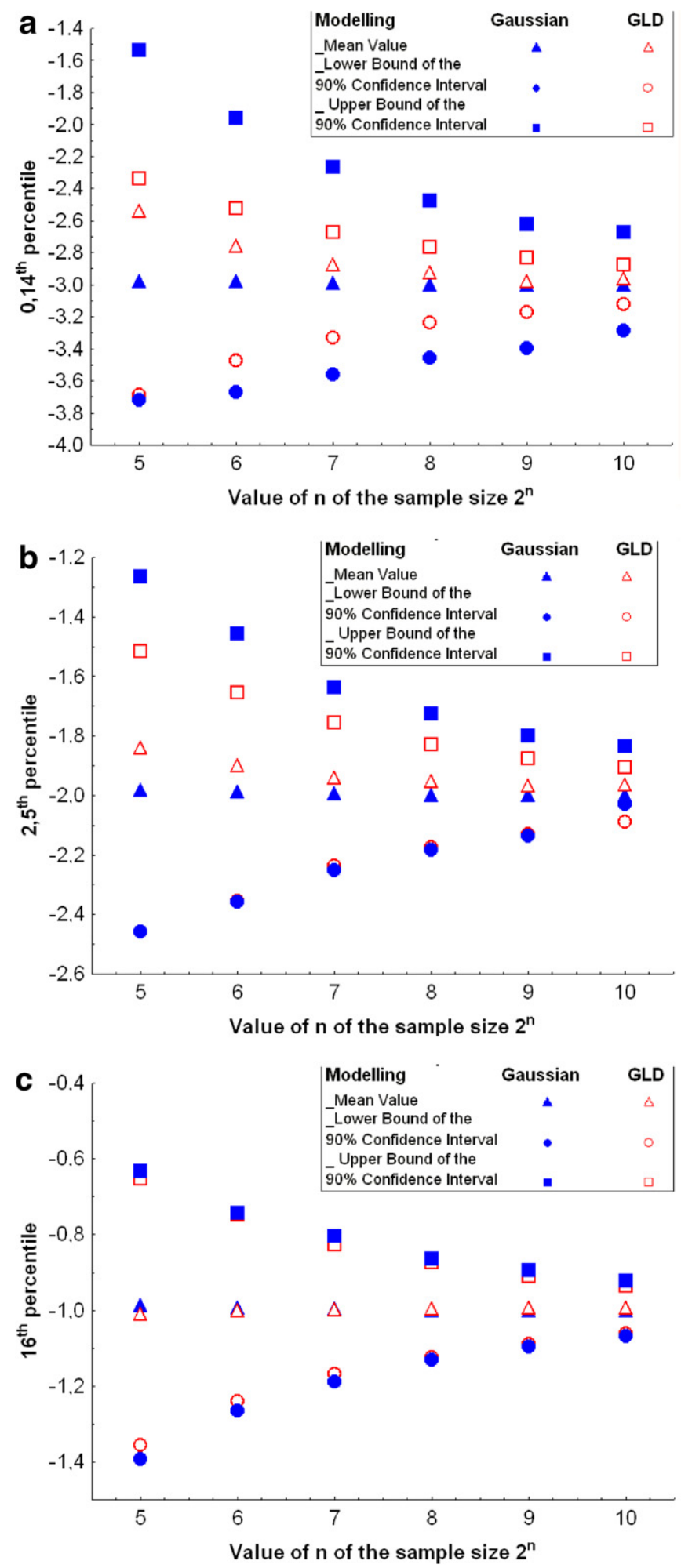

Fig. 7. Influence of the initial sample size on the evolution of the confidence intervals of the predicted SPC limits': (a) percentile 0.14th, (b) percentile 2.5 th and (c) percentile 16th - mean values for either generalized lambda distribution or Gaussian-based modelling.

\subsection{Influence of the initial population size}

The size of the initial population obviously has an effect on the accuracy of the values of the estimated parameters 
related to the statistical law retained to model the data. In this part, the influence of the initial population size on the accuracy of the SPC confidence intervals (bounded by the 0.14 th, 2.28th, 15.87th, 84.13th, 97.72th and 99.87th percentiles) is studied in the case of GLD-based modelling of data originating from a Gaussian law; i.e. in a very unfavourable case with regard to the selected model.

A 1000 samples of $2^{n}$ standard Gaussian random numbers have been generated for $n=5-10$ thanks to the Box and Muller transformation [34]. For each sample, the associated GLD has been determined using the LambdaFinder software and the 6 SPC limits have been calculated both from the initial dataset and from the analytical expression of the determined GLD percentile function. For each $n$ value, the descriptive statistics (i.e. the mean of the 1000 values and their $90 \%$ confidence intervals) of these limits have been calculated and plotted in Fig. 7.

Fig. 7 shows the evolution of the confidence intervals of only the three lowest SPC limits (i.e. 0.14th, 2.28th and 15.87th percentiles; the three upper limits present a symmetrical evolution) obtained from both the original populations of Gaussian random numbers (without modelling) and their associated GLD models. As expected, the band width of the confidence intervals related to these limits decreases as the sample size increases for both the original and modelled data. It can be noticed that the mean values of the limits predicted by using GLD-based modelling converge to the theoretical values related to the underlying Gaussian law as $n$ increases. Even for a limited number of data $\left(2^{5}=32\right)$, the mean value of the first SPC interval limit (15.87th percentile) predicted by GLD-based model- ling is almost perfect with a standard deviation lower than $1 \%$ of the theoretical value corresponding to the underlying Gaussian law. Nevertheless, Fig. 7 shows that a larger amount of initial data is required to predict accurately the 2nd and 3rd SPC lower limits (2.28th and 0.14th) since the confidence intervals of the predicted limits are very wide for $n=5$. The overall results show that starting from an initial sample of size $2^{7}$ (=128 data) the SPC limits are predicted with a precision of at least $5 \%$ on the mean value and with a standard deviation lower than $1 \%$. Such a level of accuracy is sufficient in most practical cases.

In summary, this example shows that, even in an unfavourable case, the use of GLD-based modelling requires only a hundred of initial data to estimate the SPC limits with an acceptable accuracy.

\subsection{The Lambda SPC software}

The Western Electric rules recalled in Appendix are based on the calculation of percentiles defining the median and the limits of the major confidence intervals related to the distribution under consideration. In the case of a GLD, these rules do not need to be modified since the calculation of such percentiles is particularly oversimplified from the knowledge of the related percentile function. To be compatible with the industrial need of simplicity in daily use of SPC methods, a second software has been programmed to automate completely the application of the GLD to the SPC rules in an industrial context. Called LambdaSPC and programmed in $\mathrm{C}++$ language, this second software is complementary with the LambdaFinder

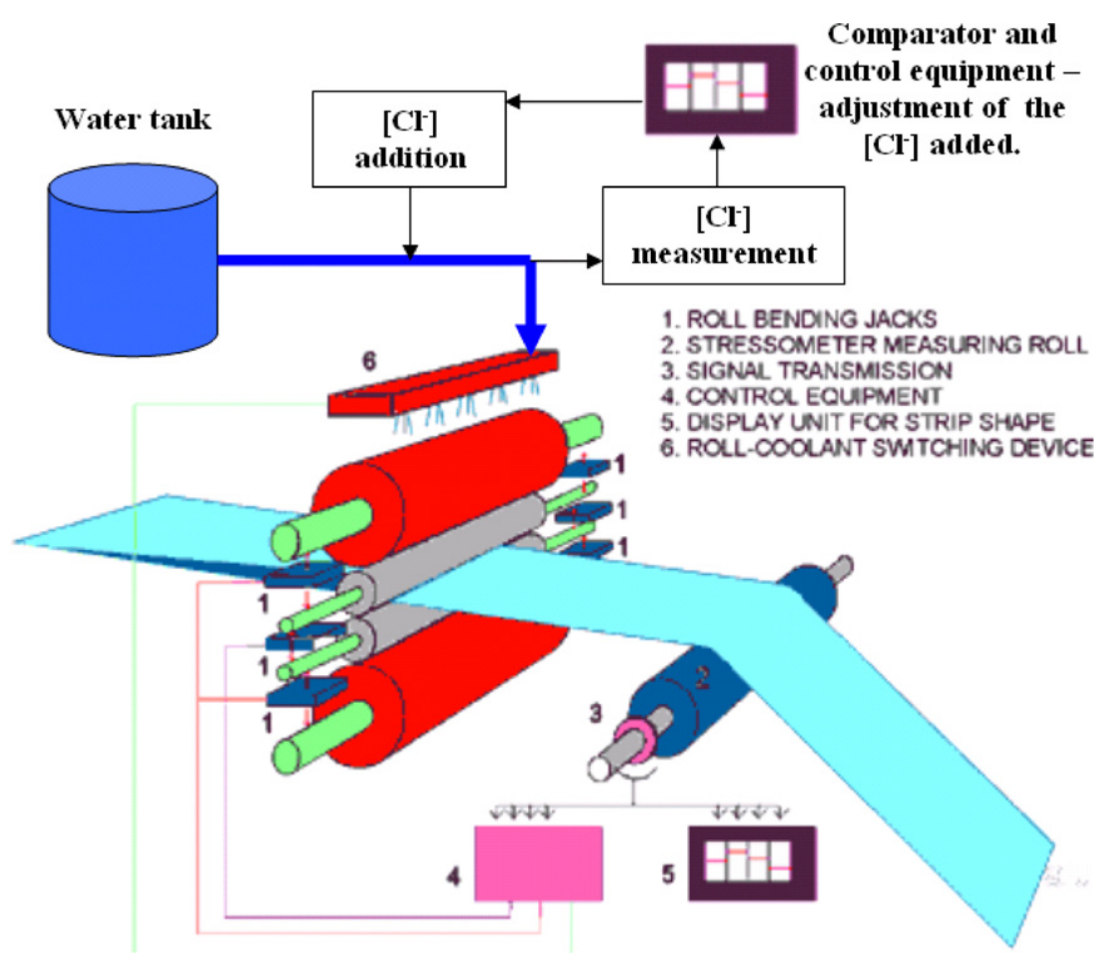

Fig. 8. Schematic process diagram of the cold rolling process with the water-oil lubricant apparatus. 
software. Indeed, in a first stage, LambdaFinder enables to determine the analytical expression of the GLD associated with the process characteristic under study and, in a second stage, LambdaSPC automatically detects any shift or abnormality in the evolution of this process over time by the application of the Western Electric rules.

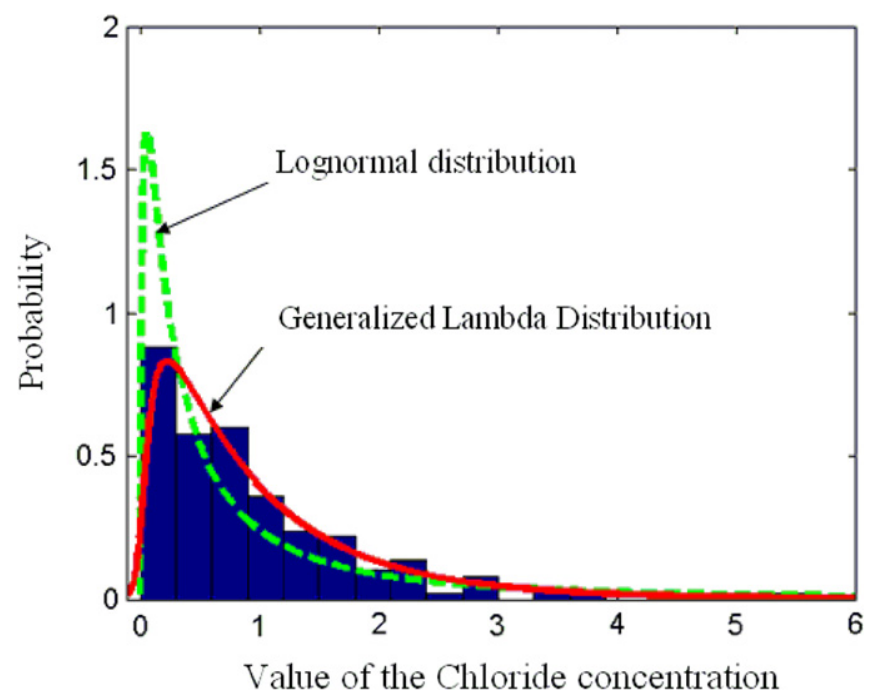

Fig. 9. Comparison between lognormal and generalized lambda distribution-based modelling of the chloride concentration probability density function.

\subsection{Example}

In order to illustrate the use and relevance of the GLD in SPC methodology, a set of industrial experimental data [35] has been studied using the two dedicated software LambdaFinder and LamdbaSPC. These experimental data consist of measures of the chloride concentration in a water-oil lubricant used during the cold rolling process of steel sheets (Fig. 8); measures recorded every hour over one week (168 values). In order to prevent post-rolling corrosion, this concentration must strictly be controlled in the sense of SPC to assert that the cold-rolling process is under control.

First of all, the LambdaFinder software is used to determine the GLD's parameters. Fig. 9 compares the GLD modelling of the dataset with parameters (0.1431, $-0.1171,-0.0037,-0.0973)$ to that obtained with a lognormal law $(\mu=-0.7088$ and $\sigma=1.4776)$. Even though the usual $\chi^{2}$ goodness-of-fit test fails to reject the Gaussian assumption for $\alpha=0.05$, it seems obvious, from this figure, that the chloride concentration does not follow a Gaussian law. Moreover the symmetry of the distribution is rejected by several simple tests [11-15], which is the reason why a lognormal law is compared to the GLD modelling.

Once the parameters obtained, the LambdaSPC software is used to draw the SPC control chart in order to detect assignable causes. Fig. 10 illustrates the control

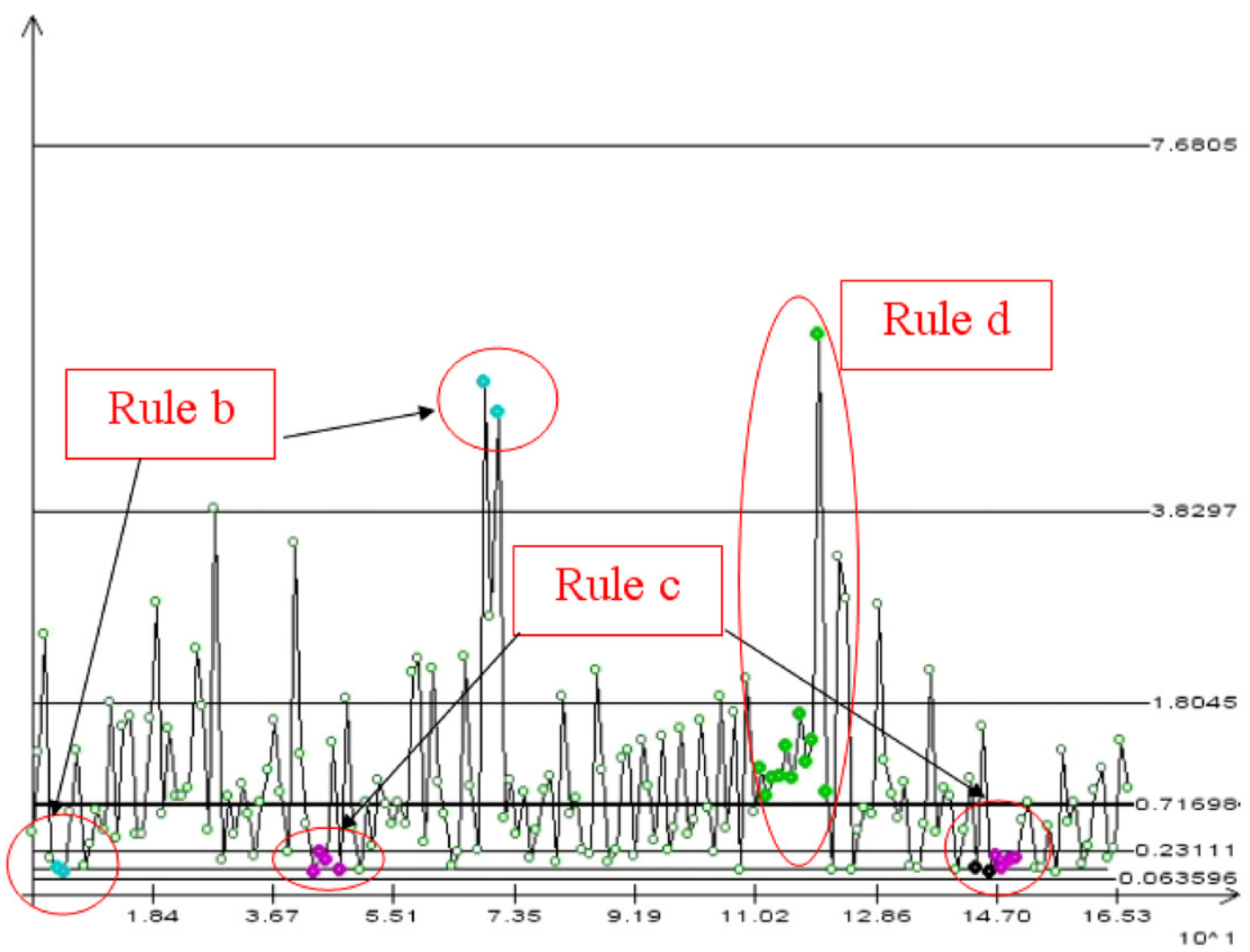

Fig. 10. Control chart obtained using the Lambda SPC software in the case of generalized lambda distribution-based modelling. Only six abnormal patterns are detected. 
Table 1

Number of process abnormalities detected both for lognormal and generalized lambda distribution-based modelling

\begin{tabular}{lll}
\hline Violated rule & $\begin{array}{l}\text { Number of occurrences } \\
\text { using a lognormal } \\
\text { based modelling }\end{array}$ & $\begin{array}{l}\text { Number of occurrences } \\
\text { using a generalized } \\
\text { lambda distribution } \\
\text { modelling }\end{array}$ \\
\hline a & 4 & 0 \\
b & 1 & 3 \\
c & 0 & 2 \\
d & 6 & 1 \\
e & 0 & 0 \\
f & 4 & 0 \\
g & 0 & 0 \\
h & 0 & 0 \\
Total & 11 & 6 \\
\hline
\end{tabular}

chart resulting from a GLD modelling. A usual SPC methodology based on a lognormal modelling would have led to eleven alert signals, whereas the lambda distribution methodology leads to only six ones over the week (Table 1). As the process is stopped when an abnormal pattern is detected in order to find an assignable cause, this means that the steel production would have been stopped unnecessarily five times in a week if the chloride concentration had been modelled using a lognormal law. Knowing the industrial cost of a production interruption, this example illustrates the economic nonsense of using the SPC methodology without an appropriate statistical modelling of the process under study.

In the previous example, the eight Western Electric rules were tested together, leading to an overall probability of a false alarm (Type I error) close to $\alpha=2.23 \%$ (see Appendix for details), which is of course an intolerably high false alarm rate for most applications. In practical SPC implementations, a choice of some of these different rules is often made, and exact calculation [36] or simulation [37] of the false alarm rates for usual combinations can be performed. A rules-selection feature is included in the LambdaSPC software in order to try different possible combinations. Nevertheless, if one wants, for a given combination of rules, to limit the overall false alarm rate to a chosen value, it is necessary to adjust these rules. Such an adjustment for multiple testing can be performed by means of a Bonferroni-type procedure [38] as advocated in the multivariate case by Alt et al. [39] or using some other method [4042]. The use of the GLD modelling has no incidence on these procedures, except that it eases the modification of the SPC limits thanks to the percentile-based definition of the GLD.

\section{Conclusions}

The limitations of classical hypothesis testing methodology with regard to the discrimination between Gaussian and non-Gaussian data were firstly highlighted through an original statistical example. As far as SPC is concerned, an alternative methodology is proposed to leave aside the nature of the unknown theoretical underlying distribution characterizing the process and hence to avoid questionable inference hypothesis. Such a methodology is based on the use of the GLD; a very flexible family of distributions that fit to a broad class of distributions including the most usual ones (uniform, lognormal, Gaussian, Weibull,...) while keeping a very accurate precision on the predicted control limits used in SPC methodology. Besides, it was shown that the minimal size of the initial dataset required to obtain an acceptable accuracy on the predicted SPC limits is only of approximately a hundred measurements when considering GLD-based modelling tested in a particularly unfavourable case. Finally, the relevance of using the GLD in SPC was illustrated treating experimental data originating from an industrial production line using the well-known Western Electric rules. Despite the mathematical complexity of determining the GLD parameters, this statistical family is made easy to use in SPC methodology thanks to the LambdaFinder and LambdaSPC software we especially developed to perform such a task. Further information on these software and a demo-version can be sent by mail by the authors upon request.

\section{Acknowledgements}

The authors would like to thank L. Minguet for his invaluable contribution to the design and programming of the LambdaFinder and LambdaSPC software, and V.Hague for the proofreading of this paper.

\section{Appendix}

The eight main rules defined in the Western Electric Handbook [1] and by Nelson $[43,44]$, under the Gaussian assumption, are the following $\left(\alpha_{i}\right.$ denotes the corresponding false alarm rate for rule $i$ ):

(a) A data point falls outside the $3 \sigma$ (with $\sigma$ the standard deviation of the reference population) control limits $\left(\alpha_{\mathrm{a}} \cong 0.27 \%\right)$.

(b) Two out of three consecutive points on the same side of the center line (which stands for the mean when the process is in-control) are between the $2 \sigma$ and $3 \sigma$ control limits or beyond the $3 \sigma$ control limits $\left(\alpha_{\mathrm{b}} \cong 0.31 \%\right)$.

(c) Four out of five consecutive points on the same side of the center line are between the $\sigma$ and $2 \sigma$ control limits or beyond the $2 \sigma$ control limits $\left(\alpha_{\mathrm{c}} \cong 0.44 \%\right)$.

(d) Nine consecutive points on the same side of the center line $\left(\alpha_{d} \cong 0.39 \%\right)$.

(e) Eight consecutive points on both sides of the center line without any point below the $1 \sigma$ control limit $\left(\alpha_{\mathrm{e}} \cong 0.01 \%\right)$.

(f) Fifteen consecutive points between $1 \sigma$ control limits on either or both sides of the center line $\left(\alpha_{f} \cong 0.32 \%\right)$.

(g) Six consecutive points steadily increasing or steadily decreasing $\left(\alpha_{g} \cong 0.28 \%\right)$. 
(h) Fourteen consecutive points alternating up and down $\left(\alpha_{b} \cong 0.29 \%\right)$.

If one considers that the eight previous rules are independent, then the overall false alarm rate $\alpha$ is given by $[45,46]$ :

$\alpha=1-\prod_{i=a}^{h}\left(1-\alpha_{i}\right)$

Nevertheless the previous formula must be considered only as an approximation of the overall false alarm rate. Indeed firstly the independence assumption is certainly not valid with the usual runs rules and additionally the $\alpha_{i}$ values are often approximated through Monte Carlo simulations, since there is not always a clear analytical expression for them.

In the case of a combination of the eight previous rules, the resulting approximation is $\alpha \cong 2.23 \%$. Using a direct Monte Carlo simulation, with no assumption about the independence between the different laws, the obtained false alarm rate (with $10^{7}$ random Gaussian datasets) is $\alpha \cong 1.37 \%$. This very high false alarm rates (an out-of-control pattern is detected every 45 or 75 measurements using respectively the previous approximation or the Monte Carlo modelling), can be reduced by modifying the previous rules using a Bonferroni-type inequality: if one wants to fix the overall false alarm rate to $\beta$, while running $\mathrm{k}$ independent tests. Then the false alarm rate of each test must be lowered to $\beta / k$ since:

$\beta \geqslant 1-\left(1-\frac{\beta}{k}\right)^{k}$

\section{References}

[1] Western Electric, Statistical Quality Handbook, AT\&T, Indianapolis, IN, 1956.

[2] J.W. Lindeberg, Eine Neue Heirletung des Exponentialgesetzs in der Warscheinlichkeitsrechnung, Mathematische Zeitschrif 15 (1922) 211-225.

[3] Y.S. Chang, K.C. Bai, Control charts for positively skewed populations with weighted standard deviations, Quality and Reliability Engineering International 17 (2001) 397-406.

[4] R.K. Pearson, Exploring process data, Journal of Process Control 11 (2001) 179-194.

[5] V. Clancy, Statistical methods in chemical analysis, Nature 4036 (1947) 339-340.

[6] T. Pyzdek, Why normal distributions aren't (all that normal), Quality Engineering 7 (1995) 769-777.

[7] M. Bigerelle, A. Iost, Traitement statistique par les Lambda Distributions et le Bootstrap généralisé des courbes de probabilité de rupture, Colloque Matériaux 2002, Tours (21-25 septembre 2002).

[8] D. Najjar, M. Bigerelle, C. Lefebvre, A. Iost, A new approach to predict the pit depth extreme value of a localized corrosion process, ISIJ 43 (5) (2003) 720-725.

[9] B. Fournier, L. Minguet, A. Niamien, N. Rupin, Les lambda distributions appliquées au SPC, unpublished report, 2003.

[10] S. Pal, Evaluation of non-normal process capability indices using generalized lambda distributions, Quality Engineering (2005) $77-85$.
[11] D.L. Hill, P.V. Rao, Tests of symmetry based on Cramer-Von Mises statistics, Biometrika 64 (1977) 489-494.

[12] I.A. Ahmad, Q. Li, Testing the symmetry of an unknown density function by the kernel method, Journal of Nonparametric Statistics 7 (1997) 279-293.

[13] M. Huskova, Hypothesis of symmetry, in: Handbook of Statistics, in: P.R. Krishnaieh, P.K. Son (Eds.), Nonparametric Methods, 4, North-Holland, New York, 1984, pp. 63-78.

[14] R.H. Randles, M.A. Fligner, G.E. Policello, D.A. Wolfe, An asymptotically distribution-free test for symmetry versus asymmetry, Journal of the American Statistical Association 369 (1980) $168-172$.

[15] M.K. Gupta, An asymptotically nonparametric test of symmetry, Annals of Mathematical Statistics 38 (1967) 849-866.

[16] M.G. Forbes, M. Guay, J.F. Forbes, Control design for first-order processes: shaping the probability density of the process state, Journal of Process Control 14 (2004) 399-410.

[17] H. Wang, Bounded Dynamic Stochastic Systems - Modelling and Control, Springer-Verlag, London, 2000.

[18] W. Albers, W.C.M. Kallenberg, Sri Nurdiati, Parametric control charts, Journal of Statistical Planning and Inference 124 (2004) 159184.

[19] Y. Lin, C. Chou, On the design of variable sample size and sampling intervals $\bar{X}$ charts under non-normality, International Journal of Production Economics 96 (2) (2005) 249-261.

[20] N.L. Johnson, Systems of frequency curves generated by methods of translation, Biometrika 36 (1949) 149-176.

[21] J.W. Tukey, The future of data analysis, Annals of Mathematical Statistics 33 (1) (1962) 1-67.

[22] J.J. Filliben, Simple and robust linear estimation of the location parameter of a symmetric distribution, Ph.D. Dissertation, Princeton University, Princeton, NJ, 1969.

[23] J.J. Filliben, The probability plot correlation coefficient test for normality, Technometrics 17 (1975) 111.

[24] B.L. Joiner, J.R. Rosenblatt, Some properties of the range in samples from Tukey's symmetric lambda distributions, Journal of the American Statistical Association 66 (1971) 394.

[25] J.S. Ramberg, B.W. Schmeiser, An approximate method for generating symmetric random variables, Communications of the ACM 15 (1972) 987-990.

[26] A. Negiz, A. Cinar, Statistical monitoring of multivariate dynamic processes with state-space models, AIChE Journal 43 (1997) 8.

[27] A. Negiz, P. Ramanauskas, A. Cinar, J.E. Schlesser, D.J. Armstrong, Modeling, monitoring and control strategies for high temperature short time pasteurization systems - 3. Statistical monitoring of product lethality ad process sensor reliability, Food Control 9 (1) (1998) 29-47.

[28] A. Tarsitano, Fitting the generalized lambda distribution to income data, COMPSTAT 2004. In: Proceedings in Computational Statistics, 16th Symposium held in Prague, Czech Republic, 2004.

[29] C.J. Corrado, Option pricing based on the generalized lambda distribution, Journal of Futures Markets 21 (2001) 213-236.

[30] A. Öztürk, R.F. Dale, A study of fitting the generalized lambda distribution to solar radiation data, Journal of Applied Meteorology 21 (7) (1982) 995-1004.

[31] Z.A. Karian, E.J. Dudewicz, Fitting Statistical Distributions: The Generalized Lambda Distribution and Generalized Bootstrap Method, CRC Press, 2000.

[32] Z.A. Karian, E.J. Dudewicz, Fitting the generalized lambda distribution to data: a method based on percentiles, Communications in Statistics: Simulation and Computation 28 (3) (1999) 793-819.

[33] M. Bigerelle, D. Najjar, B. Fournier, N. Rupin, A. Iost, Application of lambda distributions and bootstrap analysis to the prediction of fatigue lifetime and confidence intervals, International Journal of Fatigue 28 (3) (2006) 225-236.

[34] G.E.P. Box, M.E. Muller, A note on the generation of random normal deviates, Annals of Mathematical Statistics 29 (1958) 610 611. 
[35] M. Bigerelle, SPC: laminoir à froid; Analyse des produits de lubrification, rapport interne (unpublished manuscript), Arcelor, BHMB 92005, 1992.

[36] C.W. Champ, W.H. Woodall, Exact results for Shewhart control charts with supplementary run rules, Technometrics 29 (4) (1987) 260-262.

[37] E. Walker, J.W. Philopt, C. Clement, False signal rates for the Shewhart control chart with supplementary runs tests, Journal of Quality Technology 23 (3) (1991) 247-252.

[38] Bonferroni inequalities and intervals, in: S. Kotz, N.L. Johnson, C.R. Read (Eds.), The Encyclopedia of Statistical Sciences, John Wiley, New York, 1984.

[39] F.B. Alt, Multivariate quality control, in: S. Kotz, N.L. Johnson, C.R. Read (Eds.), The Encyclopedia of Statistical Sciences, John Wiley, New York, 1984.

[40] G. Nedumaran, J.J. Pignatiello, Jr., On constructing $T^{2}$ control charts for retrospective examination. Available from: $<$ http://www.eng. fsu.edu/ pigna/pdf/multret_w971-2000-01-20.pdf $>$.
[41] S. Zhang, Z. Wu, Designs of control charts with supplementary runs rules, Computers and Industrial Engineering 49 (2005) 76-97.

[42] Y. Benjamini, Y. Kling, A look at statistical process control through the $p$-values. Technical report RP-SOR-99-08. Available from: $<$ http://www.math.tau.ac.il/ kling/SPCpvalue.html $>$.

[43] L.S. Nelson, The Shewhart control chart - tests for special causes, Journal of Quality Technology 16 (4) (1984) 237-239.

[44] L.S. Nelson, Interpreting Shewhart X control chart, Journal of Quality Technology 17 (1985) 114-116.

[45] D.C. Montgomery, Introduction to Statistical Quality Control, John Wiley, New York, 1985.

[46] A.J. Duncan, Quality Control and Industrial Statistics, fifth ed., R.D. Irwin, Homewood, IL, 1986, 1123p. 